\title{
Analysis on the Relationship between Mangrove and Aquaculture in Maowei Sea Based on Object-oriented Method
}

\author{
Zixin Wei ${ }^{1, *}$ \\ ${ }^{1}$ College of Marine Science, Shanghai Ocean University, Shanghai, 201306, China
}

\begin{abstract}
Before 2000, a large number of mangrove forests in Guangxi were cut down for aqua-cultural purposes. After 2000, with the introduction of national, provincial and municipal policies on mangrove protection, this phenomenon was less frequently seen. By using the object-oriented method, referring to the spectral and texture characteristics of the ground target, and using the Landsat remote sensing images of 2000-2019, this paper extracts and analyzes the area and change of mangrove and aquaculture area in Maowei sea, Guangxi. The results show that the use of object-oriented methods can extract mangroves and aquaculture areas with higher accuracy. The area of mangroves generally shows an upward trend, with the area of aquaculture increasing first and then decreasing.
\end{abstract}

\section{Research background and significance}

The mangrove ecosystem is one of the four most productive marine ecosystems on the planet, and an important object of biodiversity protection and wetland ecological protection in the world [1]. The Guangxi Maowei Sea Autonomous Region Mangrove Forest Nature Reserve was established in 2005 by the the government of Guangxi Zhuang autonomous region. Located in Qinnan District and Qinzhou Port District of Qinzhou City, and facing the Beibu Gulf, it covers an area of 2784 hectares, of which 1892.7 hectares are mangroves. Surveys show that $1,464.1 \mathrm{hm} 2$ of mangroves in Guangxi were used for other purposes, of which $95.0 \%$ were used to build shrimp ponds from 1980 to 2000 [2]. In the early 1990 s, most of the $2557 \mathrm{hm} 2$ of aquaculture ponds along the coast of Guangxi were reclaimed from mangrove wetlands.

To overcome the limitations of such traditional methods like site investigations and pixel-based classification methods, object-oriented methods have been proposed at home and abroad to classify and extract mangrove areas and aquaculture areas using remote sensing images [3, 4]. Factors that may cause mangroves to diminish were also discussed [5-7]. However, such methods only extract a single type area, or analyze multiple factors affecting mangroves in a general way, without much analysis of the correlation between mangroves and aquaculture, an important factor, in some region in China. Therefore, employing an object-oriented classification method to extract mangroves and aquaculture areas simultaneously in Maowei Sea, Guangxi that improves accuracy, this paper provides technical support for future policy making on mangrove conservation and land use planning.

\section{General ideas and approaches}

The object-oriented method was used to extract mangrove and aquaculture areas $[4,8]$. By changing the size of segmentation with the characteristics of ground objects, this method can acquire ground objects that are optimally segmented, which made acquiring different ground objects easier. The accuracy of classification was improved since such features as ground object spectra, spatial geometric relationship, shape, and texture were used [8]. The data used in this paper are a series of remote sensing images obtained from Landsat in 2000, 2010, and 2019. With the data being preprocessed, the mangrove and aquaculture areas were extracted from the images of Maowei Sea area $\left(108^{\circ} 28^{\prime}-108^{\circ} 37^{\prime} \mathrm{E}, 21^{\circ}\right.$ $46^{\prime}-21^{\circ} 54$ ') using an object-oriented method. The mangroves in the Maowei Sea area are mainly concentrated in tidal flats. The aquaculture types are mainly offshore oyster culture, shrimp pond culture, and marine fish cage culture. Based on the situation of mangrove destruction in the area in the past, this article selected pond culture as the main object in aqua-cultural type.

\footnotetext{
* Corresponding author: fzjxwzx@126.com
} 


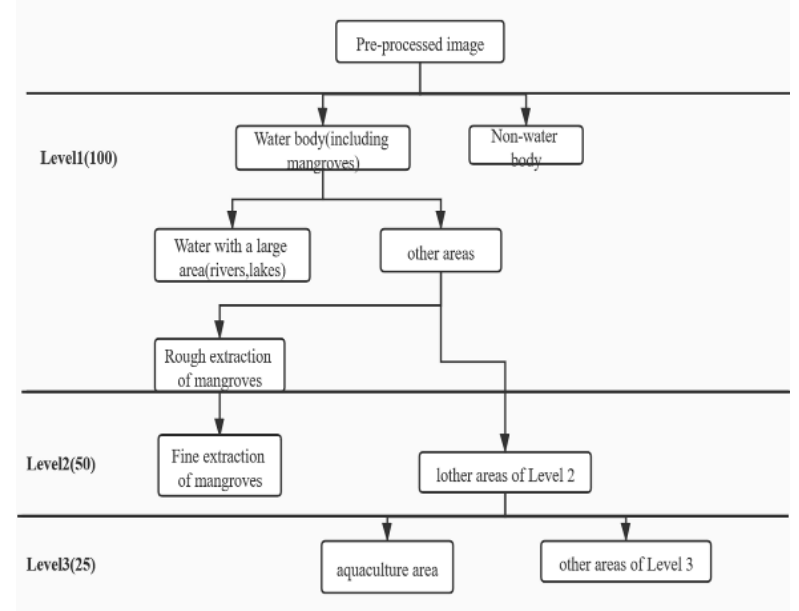

Fig. 1. Classification Extraction Flowchart

\section{Research process and analysis}

\subsection{Image segmentation}

Visual interpretation in Google Earth Pro revealed that the mangrove area was scattered in 2000, and more concentrated in 2010 and 2019, with few scattered areas located along rivers and the sea. Aquaculture areas were relatively concentrated. All in all, the segmentation scale for extracting mangroves in this region was set at 100 (Shape: 0.1, Compactness: 0.5, each band weight being 1), and 50 (Shape: 0.2, Compactness: 0.5, blue band weight being 0 , near infrared band weight being 2 ). That for the aquaculture area was set at 50, 25 (Shape: 0.1, Compactness: 0.5 , each band weight is 1 ).

\subsection{Feature extraction}

As far as mangrove areas are concerned, it could be seen from remote sensing images comprising far infrared, near infrared, and red wave band that this area presented deep green color with smooth texture. Hence, combining optical and texture features, mangrove forests were extracted with the Normalized Difference Vegetation Index (NDVI) and Normalized Difference Moisture Index (NDMI). According to conclusions drawn by Tian $\mathrm{XG}[4]$ and Chen $\mathrm{H}[9]$, texture features of mangrove forests were described as GLCM Homogeneity(all.dir.), GLCM Ang.2nd moment NIR (all.dir.), and GLCM Dissimilarity(all.dir.).

As far as aquaculture areas are concerned, remote sensing images showed that aquaculture areas in this area were similar to offshore waters as was shown in the spectrum but were different. Therefore, the modified Normalized Difference Water Index (MNDWI) and the Normalized Difference Water Index (NDWI) are used as Index of spectral characteristics of aquaculture areas. Most aquaculture areas are rectangular, which look regular, and have dikes distributed around them. According to the research by Yang HL[10], the GLCM Mean NIR(all.dir.) was adopted to distinguish aquaculture areas from tidal flats and seawater. Further, fine extraction was conducted on a small rectangular aquaculture area excluding dikes according to the rule of relative border to neighbour objects.

\subsection{Classification}

The classification process will be illustrated with 2019 as an example. The specific process of classification is given in Table 1. Different from the automatic extraction method adopted in 2019, GLCM Homogeneity NIR (all.dir.) and GLCM Ang.2nd moment NIR (all.dir.) were added to distinguish corpland from mangrove areas as the main types of ground objects in 2000 were corpland, aquaculture areas, and tidal flats. Some thresholds were modified before mangrove areas and aquaculture areas were extracted. Data from 2010 was extracted after modifying thresholds of extracted algorithm from 2019.

\subsection{Accuracy assessment and comparison}

With the object-oriented classification method, the distribution of mangroves and aquaculture areas in the study area was obtained through visual interpretation, field verification results, and literature data $[11,12]$, and was compared with automatically extracted results as reference data. It can be seen from the accuracy assessment that this method does a better job at extracting the area of mangroves, and an average one with the area of aquaculture. The area of the latter is smaller than what it is in reality, and some areas failed to be classified as aquaculture areas. This is because this study mainly discusses the relationship of changes in area between mangroves and aquaculture areas in general. It is difficult to cover all types of aquaculture in this area due to its sheer number. Therefore, only small rectangular shrimp ponds near the tidal flat area were selected. In 2000, 2010, and 2019, mangroves were extracted from the region. The overall accuracy of aquaculture areas was $89 \%, 88 \%$, and $95 \%$, and Kappa coefficients were $0.6,0.76$, and 0.89 respectively.

(Table 2) Overall, the overall accuracy of extracting regional mangroves and aqua-cultural areas using objectoriented methods is high.

In addition, the 2019 data is also used to compare the accuracy of the object-oriented classification results

(Figure 2) with the traditional pixel-based supervised classification method. The overall accuracy is both $95 \%$, but the Kappa coefficients are 0.89 and 0.79 , respectively. As the mangroves and aquaculture areas in this area are relatively concentrated, it is easy to confuse aquaculture areas with water bodies at the edge of tidal flats ( Figure 3 ) using pixel-based supervised classification methods. It can be found that objectoriented extraction methods lead to a more accurate data. 
Table 1. Rules for classifying mangrove and aquaculture areas

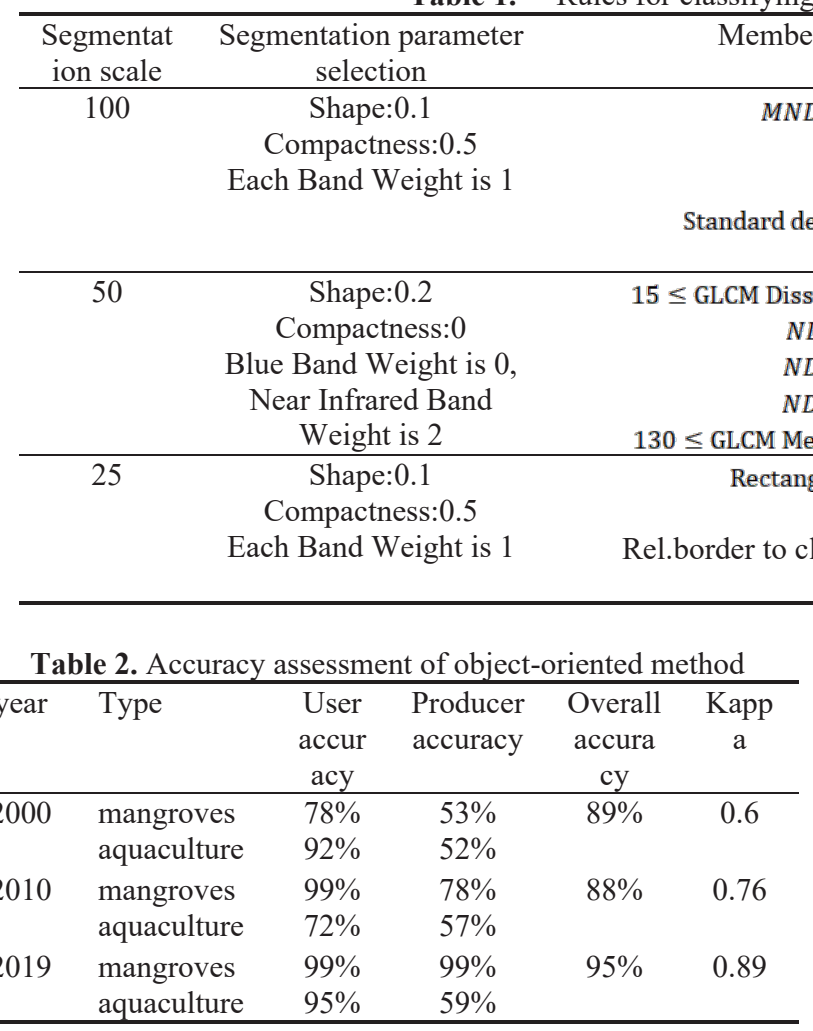

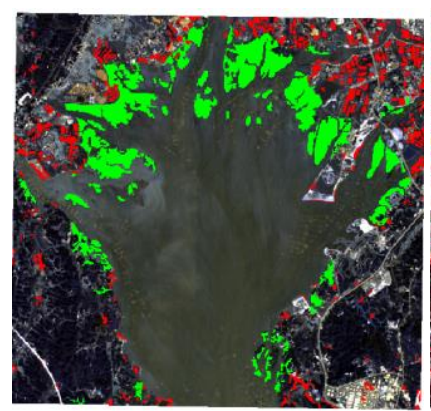

Fig. 2. Object-oriented classification

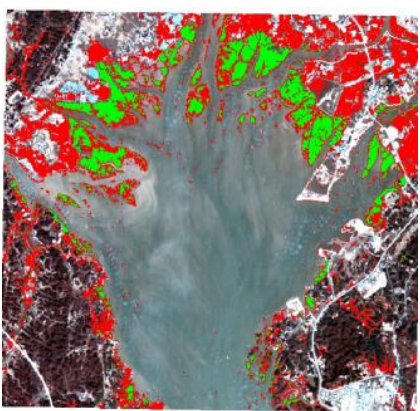

Fig. 3. Maximum likelihood supervised classification

\subsection{Analysis}

Areas that cannot be distinguished, which are few, underwent visual interpretation with Google Earth Pro before objects were merged and classified. A map was drawn after areas were determined.(Figure 4)

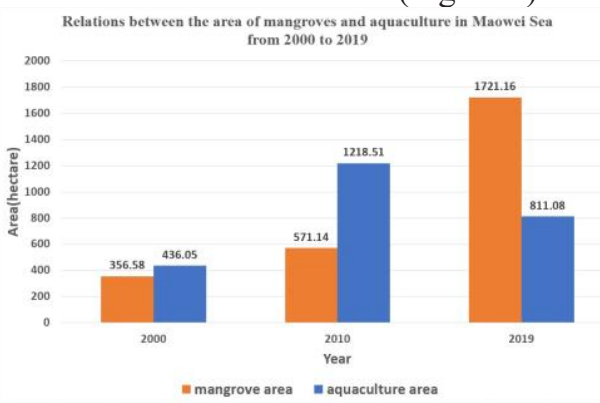

Fig. 4. Chart showing the relationship between the area of mangroves and aquaculture in Maowei Sea from 2000 to 2019

Experimental effect
Distinguish water
body(including mangrove)and
non-water body
Extraction of water with larger
areas

Extraction of mangroves

Rough extraction of aquaculture area

Fine extraction of regular and small aquaculture area

Improve the extraction of regular aquaculture areas

It was learned from further research that such situation was the result of measures to protect mangroves taken at the national and provincial level, such as the Guangxi Zhuang autonomous region and Qinzhou, a prefecture-level city. For example, the government of the autonomous region came up with the cultivation project, the third stage of mangrove planting in 2001. The Maowei Sea Mangrove Forest Conservation Area was established in 2005. The forestry department of Qinzhou has adopted measures such as increasing mangrove vegetation, promoting publicity and education about mangrove conservation, investigating cases of deforestation, and prohibiting breeding in nature reserves.

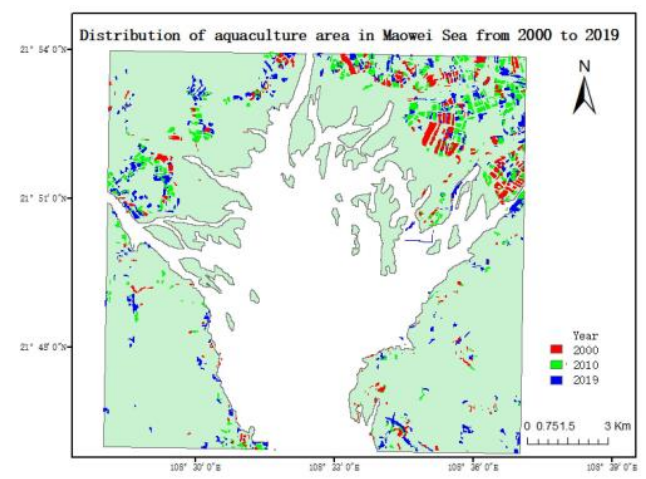

Fig. 5. Distribution of aquaculture area in Maowei Sea from 2000 to 2019 .

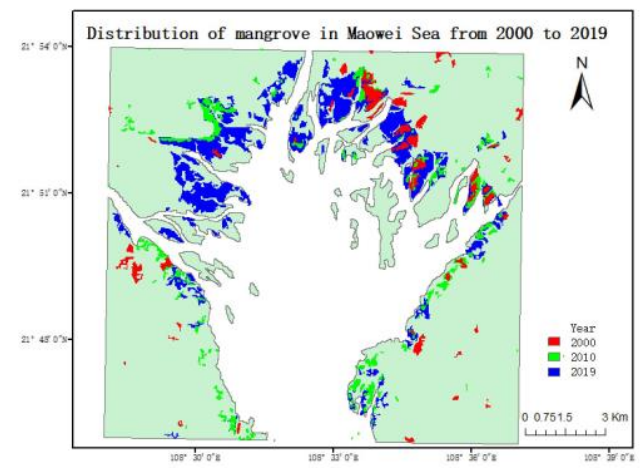

Fig. 6. Distribution of mangroves in Maowei Sea from 2000 to 2019 
The area of mangroves in this research area increased slowly from 356.58 hectares in 2000 before experiencing a rapid growth to 1721.16 hectares in 2019. Scattered parts of mangroves in tidal flats expanded gradually to cover almost the tidal flats. The aquaculture areas rapidly expanded from 436.05 hectares in 2000 to 1218.51 hectares in 2010, and dropped to 811.08 hectares in 2019 due to proper control. If one looks at mangrove areas and aquaculture areas that underwent changes, one would discover that mangroves that have experienced less severe damage since 2000 have been used in aquaculture in the Maowei Sea area, while tidal flats extending to sea areas in tidal flats witnessed mangroves be vigorously planted.

\section{Conclusion}

In this paper, an object-oriented classification method is used to achieve targeted extraction of mangroves and aquaculture areas in the Maowei Sea, with enhanced extraction accuracy. The results show that chopping down mangroves for aqua-cultural purposes have been rarer in the Maowei Sea area since 2000. However, due to the complexity of aqua-cultural types in the region, this article didn't analyze all aqua-cultural types. In subsequent studies, specific extraction and analysis can be performed for specific aqua-cultural types in the region.

\section{References}

1. Kathiresan, K. and B.L. Bingham, Biology of mangroves and mangrove ecosystems. Advances in marine biology, (2001) 40: p. 84-254.

2. Department of Forest Resources Management, State Forestry Administration, China Mangrove Resources Survey Report. (2002)

3. $\mathrm{Xu} \mathrm{S}$, et al., Research on remote sensing extraction of object-based marine aquaculture models. Surveying and spatial geographic information, (2018) 41 (05): p. 110-112.

4. Tian XG, Zhang XJ, and Zhang YH, Objectoriented Mangrove Information Extraction. Marine Surveying, (2007) 02: p. 41-44.

5. Jia, MM, et al., Monitoring loss and recovery of mangrove forests during 42 years: The achievements of mangrove conservation in China. International journal of applied earth observation and geoinformation, (2018) 73: p. 535-545.

6. Guimarães, A.S., et al., Impact of aquaculture on mangrove areas in the northern Pernambuco Coast (Brazil) using remote sensing and geographic information system. Aquaculture Research, (2010) 41(6): p. 828-838

7. Herbeck, L.S., et al., Decadal trends in mangrove and pond aquaculture cover on Hainan (China) since 1966: mangrove loss, fragmentation and associated biogeochemical changes. Estuarine, Coastal and Shelf Science, (2020) 233: p. 106531.
8. Wang XX, Object-oriented Coastal Aquaculture Pattern Recognition. (2011) Guangzhou University.

9. Chen H, Application of Gray Level Co-occurrence Matrix in Texture Characteristic Analysis of Remote Sensing Image of Cultivated Land, in The Second National Conference on Remote Sensing Technology of Land and Resources. (2006) Changchun.

10. Yang HL, Fu J, and Gu DQ, Application of texture analysis method to classification of coastal features. Advances in Marine Science, (2011) 29 (02): p. 196-204.

11. Ma YM, et al., Classification and coverage estimation of mangrove forests in Maowei Sea, Guangxi based on GF-2 images. Marine Sciences, (2019) 43 (06): p. 60-70.

12. Jia, MM., et al., Landsat-based estimation of mangrove forest loss and restoration in Guangxi province, China, influenced by human and natural factors. IEEE Journal of Selected Topics in Applied Earth Observations and Remote Sensing, (2014) 8(1): p. 311-323. 